\title{
Photoemission study of electronic structure of the half-metallic ferromagnet $\mathrm{Co}_{3} \mathrm{Sn}_{2} \mathrm{~S}_{2}$
}

\author{
M. Holder, ${ }^{1}$ Yu. S. Dedkov,,${ }^{1,2}$ A. Kade, ${ }^{1}$ H. Rosner,${ }^{3}$ W. Schnelle, ${ }^{3}$ A. Leithe-Jasper, ${ }^{3}$ \\ R. Weihrich, ${ }^{4}$ and S. L. Molodtsov ${ }^{1,5}$ \\ ${ }^{1}$ Institut für Festkörperphysik, Technische Universität Dresden, 01062 Dresden, Germany \\ ${ }^{2}$ Fritz-Haber-Institut der Max-Planck-Gesellschaft, 14195, Berlin, Germany \\ ${ }^{3}$ Max-Planck-Institut für Chemische Physik fester Stoffe, 01187 Dresden, Germany \\ ${ }^{4}$ Institut für Anorganische Chemie, Universität Regensburg, 93040 Regensburg, Germany \\ ${ }^{5}$ Institute of Physics, St. Petersburg State University, 198904 St. Petersburg, Russia \\ (Received 10 January 2009; published 26 May 2009)
}

\begin{abstract}
Surface electronic structure of polycrystalline and single-crystalline samples of the half-metallic ferromagnet $\mathrm{Co}_{3} \mathrm{Sn}_{2} \mathrm{~S}_{2}$ was studied by means of angle-resolved and core-level photoemissions. The experiments were performed in temperature regimes both above and below a Curie temperature of $176.9 \mathrm{~K}$. The spectroscopic results are compared to local-spin density approximation band-structure calculations for the bulk samples. It is found that the surface sensitive experimental data are generally reproduced by the bulk computation suggesting that the theoretically predicted half-metallic properties of $\mathrm{Co}_{3} \mathrm{Sn}_{2} \mathrm{~S}_{2}$ are retained at the surface.
\end{abstract}

DOI: 10.1103/PhysRevB.79.205116

PACS number(s): $71.20 .-\mathrm{b}$

\section{INTRODUCTION}

The concept of "half-metallic ferromagnetism" was introduced by de Groot et al. ${ }^{1}$ for Heusler phases that are considered to be highly relevant for spin-dependent manipulation of charge carriers. ${ }^{2}$ Spin-polarized band-structure calculations for NiMnSb reveal metallic properties for the spinmajority electrons while the spin-minority states possess a band gap at the Fermi energy $\left(E_{F}\right)$. These theoretical predictions were, however, controversially inspected by spectroscopic experiments. While only $50 \%$ spin polarization of the Fermi-energy electrons was measured by spin-resolved photoemission (PE) ${ }^{3}$ a spin-resolved inverse PE study shows that the polarization at $E_{F}$ can approach $100 \% .{ }^{4}$ The obtained discrepancy was explained by possible segregation phenomena that may lead to considerable deviations of the surface as compared to bulk stoichiometry. ${ }^{3-5}$ On the other hand, due to reduced coordination even stoichiometric surfaces may exhibit an electronic structure that is very different compared to the bulk. ${ }^{6}$ But driven by miniaturization of electronic devices, in particular electronic structure at the surface as probed by spectroscopic techniques became of highest importance for applications.

A half-metallic ferromagnetic behavior was recently predicted by Weihrich and Anusca ${ }^{7}$ for the shandite $\mathrm{Co}_{3} \mathrm{Sn}_{2} \mathrm{~S}_{2}$. The term shandite will be used in this paper for all compounds isostructural to $\mathrm{Ni}_{3} \mathrm{~Pb}_{2} \mathrm{~S}_{2}$. From band-structure calculation they found that a ferromagnetic transition in this compound is accompanied by the formation of a band gap in the spin-minority direction. Since the Fermi level lies within this gap, $\mathrm{Co}_{3} \mathrm{Sn}_{2} \mathrm{~S}_{2}$, which is metallic in nonmagnetic state, becomes insulating for spin-minority states at a Curie temperature $\left(T_{C}\right)$ of $176.9 \mathrm{~K}^{8}$ From a classification given by Coey and Venkatesan ${ }^{9} \mathrm{Co}_{3} \mathrm{Sn}_{2} \mathrm{~S}_{2}$ can be assigned to type $\mathrm{I}_{A}$ halfmetallic ferromagnets.

Measurements of magnetic susceptibility ${ }^{8,10}$ confirm the results of the bulk band-structure calculation. It was found that the ferromagnetism in $\mathrm{Co}_{3} \mathrm{Sn}_{2} \mathrm{~S}_{2}$ is rather sensitive to structural deviations as demonstrated by substituting Co by
$\mathrm{Ni}$. Only a few percent of $\mathrm{Ni}$ impurities decrease rapidly the $T_{C}$ in $\left(\mathrm{Co}_{1-x} \mathrm{Ni}_{x}\right)_{3} \mathrm{Sn}_{2} \mathrm{~S}_{2}$. Above $x \simeq 0.2$ the compound is no more ferromagnetic. ${ }^{10}$ This experimental finding is in accordance with band-structure calculations performed for $\mathrm{Ni}_{3} \mathrm{Sn}_{2} \mathrm{~S}_{2}$ by Weihrich et al. ${ }^{11}$ In this shandite, the energy gap in exchange-split transition-metal $3 d$ states is shifted to higher binding energy (BE) as compared to its position in $\mathrm{Co}_{3} \mathrm{Sn}_{2} \mathrm{~S}_{2}$. Thus, the transition to ferromagnetism is suppressed. Another important issue reported in Ref. 7 is the influence of $\mathrm{Sn}$ and $\mathrm{S}$ atoms on electronic properties of $\left(\mathrm{Co}_{1-x} \mathrm{Ni}_{x}\right)_{3} \mathrm{Sn}_{2} \mathrm{~S}_{2}$. Although $\mathrm{Sn}$ and $\mathrm{S}$ originating contributions to valence-band (VB) density of states (DOS) are negligible, particularly S-Sn-S bonds along (111) direction of the crystals are of high importance for the gap formation.

In this contribution we focus on surface electronic structure of $\mathrm{Co}_{3} \mathrm{Sn}_{2} \mathrm{~S}_{2}$. Experimental results measured by means of angle-resolved and core-level PE for single crystals, both above and below $T_{C}$, are compared with the corresponding data taken for polycrystalline samples. The spectroscopic results are compared to the local-spin density approximation (LSDA) bulk band-structure calculations performed for the nonmagnetic and ferromagnetic configurations. It is found that the rather surface sensitive experimental data are generally reproduced by the bulk computation. An enhancement of the Fermi-energy signal measured below $T_{C}$ is in accordance with the predicted magnetic band splitting resulting in a downward energy shift of the spin-majority bands that were unoccupied in the nonmagnetic sample. Spin-polarized spectroscopic experiments are necessary to quantify expected spin polarization of the Fermi-energy electrons in the surface region of $\mathrm{Co}_{3} \mathrm{Sn}_{2} \mathrm{~S}_{2}$.

\section{EXPERIMENTAL AND THEORETICAL DETAILS}

\section{A. Samples}

Stoichiometric single crystals have been grown by a modified vertical Bridgman technique. For this purpose polycrystalline $\mathrm{Co}_{3} \mathrm{Sn}_{2} \mathrm{~S}_{2}$ was placed in a tipped glassy carbon 
crucible. The crucible was inserted into a tantalum tube which was closed by arc welding. Synthesis and handling steps were carried out in an argon gas glove box system $\left(p_{\mathrm{O}_{2}}\right.$, $\left.p_{\mathrm{H}_{2} \mathrm{O}}<0.1 \mathrm{ppm}\right)$. The closed tantalum tube was then sealed under vacuum in a quartz tube which was suspended by a Kanthal thread from the top to the hot zone of a resistance heated vertical tube furnace. The sample was slowly heated up to $1000{ }^{\circ} \mathrm{C}$, kept there for $6 \mathrm{~h}$, and slowly cooled within $72 \mathrm{~h}$ to $800{ }^{\circ} \mathrm{C}$. After these steps the furnace was turned off and the samples were cooled down to ambient temperature. The crystal was removed from the glassy carbon crucible by careful mechanical fragmentation of the crucible. The grown crystals of typical lengths of 10 and $1 \mathrm{~cm}$ in diameter were cut, polished, and inspected by optical metallography. They were found to contain several large grains which could be mechanically separated. The chemical composition was checked by energy dispersive x-ray analysis. The lattice parameters which were obtained from refinement of powder $\mathrm{x}$-ray diffraction data of a crushed piece of the crystal are in agreement with the literature. ${ }^{12}$

\section{B. Photoelectron spectroscopy}

High-resolution angle-resolved and core-level PE studies were carried out at the Berliner Elektronenspeicherring für Synchrotronstrahlung (BESSY) (beamline UE112-PGM-1) and in the laboratory of the Dresden University of Technology. The measurements were performed in ultrahigh-vacuum systems consisting of two distinct chambers for preparation and analysis. The PE spectra ( $h \nu=21.2$, 40.8, 70, 200, 880, and $1486.6 \mathrm{eV}\left[\mathrm{Al} K_{\alpha}\right]$ ) were recorded with $180^{\circ}$ hemispherical electron-energy analyzers (PHOIBOS 150 at BESSY and Scienta 2002 in the laboratory) with energy resolution of 150 and $400 \mathrm{meV}$ at full width at half maximum (FWHM) in the photon-energy region below 200 and above $880 \mathrm{eV}$, respectively. The angle-resolved PE data in the region of the Fermi energy were analyzed applying deconvolution with a Gaussian accounting for the finite experimental resolution. The measured S $2 p$ core-level PE spectra were simulated exploiting the WIN-FIT program. ${ }^{13} \mathrm{PE}$ peaks originating from $\mathrm{S}$ atoms in different chemical environments were least squares fitted with Voigt-shaped subspectra superimposed by a steplike integral background.

The analyzers were fixed while the vertically positioned $\mathrm{Co}_{3} \mathrm{Sn}_{2} \mathrm{~S}_{2}$ samples were rotated, changing the incidence angle of photons as well as the polar angle of emitted photoelectrons. In order to get clean sample surfaces as necessary for PE experiments, the single-crystalline species were cleaved in situ by means of ceramic or metallic sticks glued at the crystal surfaces in a vacuum better than 3 $\times 10^{-10}$ mbar. The polycrystalline samples were scraped with a diamond file at a pressure better than 8 $\times 10^{-10}$ mbar. Base pressure during the measurements was always in the range of $1 \times 10^{-10}$ mbar. The samples were studied at room temperature and $40 \mathrm{~K}$, which is above and below $T_{C}$.

Surface contaminations were monitored by tracing $\mathrm{C}$ and O $1 s$ core-level signals in photoemission spectra of the species. The Fermi-level energy position of the analyzers were calibrated applying copper polycrystalline foils in electrical contact with the samples.

\section{Band-structure calculations}

Band-structure calculations were performed using the full-potential local-orbital minimum basis code FPLO (version 5.00-19, Ref. 14) within the local-spin density approximation. In the scalar relativistic calculation the exchange and correlation potential of Perdew and Wang ${ }^{15}$ was employed. A basis set was constructed from a combination of Co $3 s$ and $3 p$, and Sn $4 s, 4 p$, and $4 d$ semicore states as well as Co $4 s$, $4 p$, and $4 d$, Sn $5 s, 5 p$, and $5 d$, and $\mathrm{S} 3 s, 3 p$, and $3 d$ valence states. All lower lying states were treated fully relativistic as core states. The inclusion of the semicore states was forced by their non-negligible overlap due to the large extension of their wave functions. The formally unoccupied $\mathrm{Sn} 5 d$ and S $3 d$ states were included to improve the completeness of the basis set. The extension of the valence orbitals was controlled by an additional confining potential $\left(r / r_{0}\right)$ with the parameter $r_{0}$ optimized with respect to the total energy. ${ }^{16} \mathrm{~A}$ dense mesh of $8000 \mathbf{k}$ points in the Brillouin zone (BZ) has been applied to ensure accurate DOS and band-structure information. The calculations were carried out for the experimentally determined structural data (see next section).

\section{RESULTS AND DISCUSSION}

\section{A. Sample characterization}

\section{Surface structure}

The crystal structure of the shandite $\mathrm{Co}_{3} \mathrm{Sn}_{2} \mathrm{~S}_{2}$ is discussed in detail in Refs. 11 and 17. It is denoted by trigonalrhombohedral symmetry of space-group $166(R \overline{3} m)$, a lattice parameter $a=5.3754 \AA$, and an axis angle of $59.91^{\circ}$. According to the trigonal axes, cobalt atoms occupy Wyckoff position $3 e$, tin atoms are placed in $1 a$ and $1 b$, and sulfur atoms reside in position $2 c$ with $z(\mathrm{~S})=0.216$. The crystal structure of $\mathrm{Co}_{3} \mathrm{Sn}_{2} \mathrm{~S}_{2}$ is displayed in Fig. 1(a).

Owing to a quasilayered arrangement of atoms in $\mathrm{Co}_{3} \mathrm{Sn}_{2} \mathrm{~S}_{2}$, cleaved surfaces of measured single crystals are always perpendicular to [111] direction. Thus, three surface terminations are possible in the experiment: Sn atoms surrounded by either (i) six Co or (ii) six $\mathrm{Sn}$ atoms, and (iii) $\mathrm{S}$ atoms surrounded by six $\mathrm{S}$ atoms. Since all (111) planes mentioned above exhibit close-packed atomic structures, interpretation of PE spectra in terms of hexagonal symmetry, as indicated in Fig. 1(a), is justified.

The quality of the surface was checked by low energy electron diffraction (LEED). Obtained sharp images are displayed in Figs. 1(b)-1(d). The hexagonal structure of (111) surface becomes visible at 60 and $80 \mathrm{eV}$ primary electronbeam energies. Thereby, the intensity of the LEED spots is strongly electron-energy modulated (compare to $50 \mathrm{eV}$ pattern) as expected from the multiatom basis of the crystal.

\section{Core-level photoemission}

To characterize electronic properties of the singlecrystalline $\mathrm{Co}_{3} \mathrm{Sn}_{2} \mathrm{~S}_{2}$ samples, first a series of their core lev- 
(a)
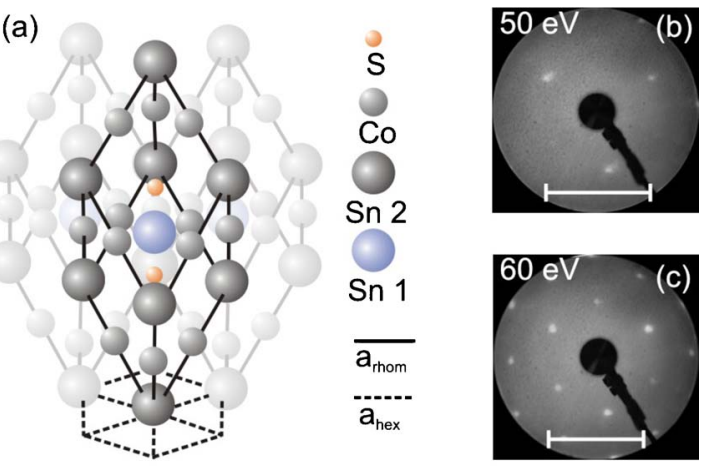

(e)

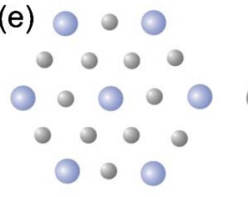

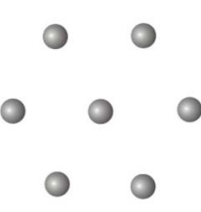

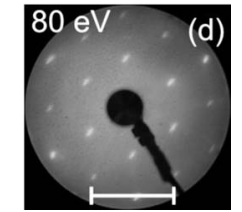

FIG. 1. (Color online) (a) Crystal structure of $\mathrm{Co}_{3} \mathrm{Sn}_{2} \mathrm{~S}_{2}$ with rhombohedral basis. Axes of the hexagonal basis are also indicated. [(b)-(d)] LEED images obtained from (111) surface at different primary electron-beam energies. Bars denote scaling of the reciprocal lattice with electron-beam energy. (e) Two possible surface terminations [(i) and (ii), see text] obtained in experiments.

els was measured and compared with corresponding data taken for polycrystalline specimens. The core-level PE spectra of the polycrystals were acquired upon $\mathrm{Al} K_{\alpha}$ excitation while the single crystals were probed with either 200 or 880 $\mathrm{eV}$ photon energy.

The obtained spectra are shown in Fig. 2. The measured binding energies are compared to results reported in the literature for a number of related compounds in order to gain information about electronic configurations and bonding character in $\mathrm{Co}_{3} \mathrm{Sn}_{2} \mathrm{~S}_{2}$. For the isostructural
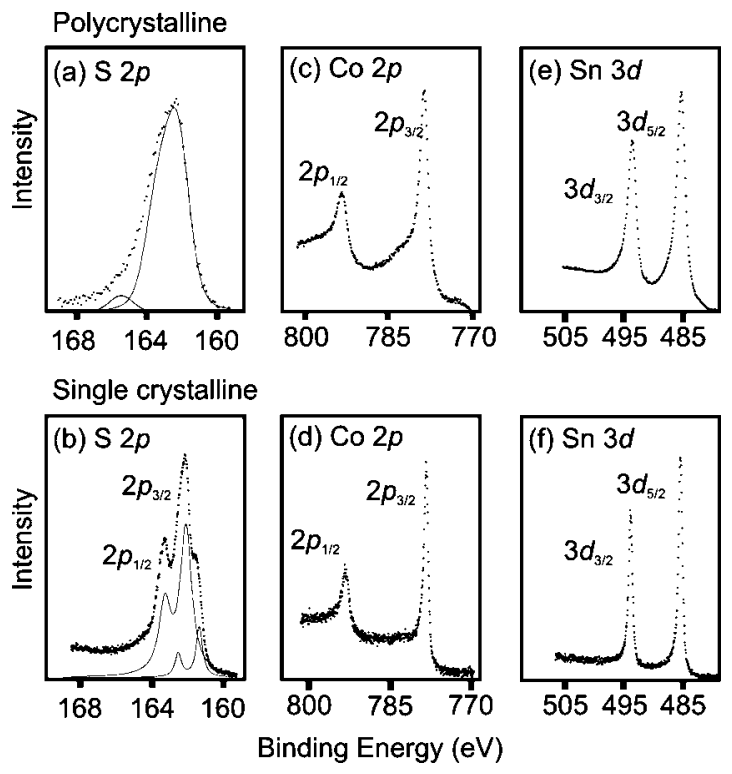

FIG. 2. Core-level PE spectra measured for polycrystalline and single-crystalline samples. Results of the least-squares fit analysis are shown by thin lines underneath the $\mathrm{S} 2 p$ spectra.

compound $\mathrm{Ni}_{3} \mathrm{Sn}_{2} \mathrm{~S}_{2}$, the electronic configuration $\left(\mathrm{Ni}^{0}\right)_{3}\left[\mathrm{Sn}(1)^{2+}\right]\left[\mathrm{Sn}(2)^{2+}\right]\left(\mathrm{S}^{2-}\right)_{2}$ was derived from Mößbauer and core-level PE experiments. ${ }^{18}$ Note that the charge of the $\mathrm{S}^{2-}$ ions is completely compensated by the $\mathrm{Sn}$ atoms while the $\mathrm{Ni}$ atoms remain neutral.

Indeed $\mathrm{S}^{2-}$ configuration in $\mathrm{Co}_{3} \mathrm{Sn}_{2} \mathrm{~S}_{2}$ is confirmed by measured $\mathrm{BE}$ of $162.46 \mathrm{eV}$ for the $\mathrm{S} 2 p$ state [main component in Figs. 2(a) and 2(b)]. In $\mathrm{Co}_{3} \mathrm{Sn}_{2} \mathrm{~S}_{2}$, the core level is slightly shifted toward lower binding energy compared to $\mathrm{Ni}_{3} \mathrm{Sn}_{2} \mathrm{~S}_{2}(162.8 \mathrm{eV}){ }^{18}$ This shift is not related to an ionic bonding caused by possible charge transfer from the transition metal to the sulfur. Co may provide one $3 d$ valence electron less than $\mathrm{Ni}$ and, therefore, the charge density on the sulfur site is expected to be larger in $\mathrm{Ni}_{3} \mathrm{Sn}_{2} \mathrm{~S}_{2}$. Thus, the covalent bonding, accompanied by a decrease in the bond length (Ni-S: $2.191 \AA$, Co-S: $2.178 \AA$ ) or different screening of the PE core-hole excitation might cause the observed $\mathrm{BE}$ behavior. A similar situation is encountered in transitionmetal pyrites. When going from $\mathrm{Co}\left(\mathrm{S}_{2}\right)^{2-}$ to $\mathrm{Ni}\left(\mathrm{S}_{2}\right)^{2-}$ the binding energy of the $\mathrm{S} 2 p$ core level is increased by 0.1 $\mathrm{eV} .{ }^{19}$ This fact supports the above conclusion that particularly the interplay between the transition-metal and sulfur atoms is responsible for the observed $\mathrm{BE}$ variation. In $\mathrm{CoS}$ with a $\mathrm{S}^{2-}$ configuration the $\mathrm{S} 2 p \mathrm{PE}$ signal is found at 161.8 $\mathrm{eV}$ binding energy. ${ }^{20}$ Here, the $\mathrm{S}$ atom is coordinated by six Co atoms in a trigonal prismatic arrangement, contrary to antiprismatic configuration in $\mathrm{Co}_{3} \mathrm{Sn}_{2} \mathrm{~S}_{2}$.

Besides the main contribution to the $\mathrm{S} 2 p$ spectra discussed above, which is related to the $\mathrm{S}^{2-}$ configuration, an additional weak component at $165.5 \mathrm{eV}$ binding energy is found for the polycrystalline samples [see Fig. 2(a)]. According to Ref. 21 this component stems from $\mathrm{S}^{0}$ atoms and can be assigned to nonstoichiometric sulfur. For the singlecrystalline species, S $2 p$ spectra reveal fine structure due to well-resolved spin-orbit splitting that is smeared out in lower quality polycrystalline samples owing to chemically inequivalent positions of the $\mathrm{S}$ atoms. Apart from the main doublet subspectrum, a second doublet shifted by $0.7 \mathrm{eV}$ to lower BE is monitored [Fig. 2(b)]. This minor component is interpreted as a surface-derived contribution. The reduced coordination number at the surface causes band narrowing that, in accordance with our calculations, results in an increased density of electronic states immediately below the Fermi energy. The constraint that the solid has to be charge neutral leads then to the shift of surface component to lower binding energy. The fact that the bulk $\mathrm{S}$ subspectrum is characterized by much higher intensity evidences that the cleaved surface is in any case not primarily $\mathrm{S}$ terminated. It leaves mainly the (i) and (ii) possibilities for surface termination [Fig. 1(e)]. Note that the $\mathrm{S}^{0}$ atoms do not noticeably contribute to the PE intensity in Fig. 2(b) pointing at good quality of the single-crystalline $\mathrm{Co}_{3} \mathrm{Sn}_{2} \mathrm{~S}_{2}$ samples measured in the present study.

The binding energy of the Co $2 p_{3 / 2}$ level was measured to be $778.4 \mathrm{eV}$ for both polycrystalline and single-crystalline samples [Figs. 2(c) and 2(d)]. This value is close to the ones observed in $\mathrm{CoS}_{2}(778.1 \mathrm{eV})$ and $\mathrm{CoSe}_{2}(778.3 \mathrm{eV})$, and would support a $\mathrm{Co}^{2+}$ valence state. ${ }^{19}$ In the cobalt lower valent CoSe compound, the Co $2 p_{3 / 2}$ binding energy is 778.7 $\mathrm{eV}^{22}$ However, the length of Co-S bonds in $\mathrm{Co}_{3} \mathrm{Sn}_{2} \mathrm{~S}_{2}$ indi- 
cates covalency. On the other hand, the observed peak separation of $15.1 \mathrm{eV}$ between $2 p_{3 / 2}$ and $2 p_{1 / 2}$ states points at the metallic Co-Sn bonds, contrary to a separation of $16 \mathrm{eV}$ in $\mathrm{Co}^{2+}$ compounds. Thus, although pure ionic bonding should be ruled out, the situation is not as clear as in $\mathrm{Ni}_{3} \mathrm{Sn}_{2} \mathrm{~S}_{2}$ and some charging of the Co atoms is expected.

Analyzing line shapes of the Co core-level spectra, no satellite structures as reported in Refs. 23 and 24 are observed. In these studies the satellite structures were assigned to atomiclike multiplets well known for correlated materials where PE core-hole interacts with partly filled localized shells of $d$ or $f$ electrons. ${ }^{20}$ This multiplet structure of the $2 p_{3 / 2}$ core level $(\sim 778.0 \mathrm{eV} \mathrm{BE})$ was not observed in Co metal $^{20}$ pointing at purely itinerant character of $3 d$ electrons there. Our data show that also in $\mathrm{Co}_{3} \mathrm{Sn}_{2} \mathrm{~S}_{2}$ all valence states are rather extended which justifies our theoretical description of the electronic structure within the LSDA approach. We may assume that the satellite structure measured in Ref. 24 might be related to possible sample oxidation as discussed by Galtayries and Grimblot. ${ }^{20}$

The line shape of the Co $2 p$ core-level spectra measured for polycrystalline samples is clearly asymmetric with a tail extending in the region of low binding energies [see Fig. 2(c)]. This observation can be explained by photoelectrons scattered inelastically on impurities and grain boundaries and signals stemming from Co atoms occupying nonstoichiometric positions in $\mathrm{Co}_{3} \mathrm{Sn}_{2} \mathrm{~S}_{2}$ polycrystals. This asymmetry is strongly reduced in PE spectra measured for the high-quality single-crystalline species [Fig. 2(d)].

From the data obtained by ${ }^{119} \mathrm{Sn}$ Mößbauer spectroscopy in Ref. 18 a valence state 2+ was concluded for the Sn atoms in $\mathrm{Ni}_{3} \mathrm{Sn}_{2} \mathrm{~S}_{2}$. Similar measurements done by Weihrich et al. ${ }^{25}$ on $\mathrm{Co}_{3} \mathrm{Sn}_{2} \mathrm{~S}_{2}$ point at partly filled $\mathrm{Sn} 5 s$ shells. In our PE measurements (not shown) the $\mathrm{Sn} 4 d_{5 / 2}$ peak in $\mathrm{Co}_{3} \mathrm{Sn}_{2} \mathrm{~S}_{2}$ was found at $24.6 \mathrm{eV} \mathrm{BE}$. This energy lies in the range known for Sn chalcogenides (24.5-24.8 eV, Ref. 26) and deviates considerably from the value reported for elemental Sn (24.0 eV, Ref. 27). Oxidation of Sn would shift the $4 d$ peak to higher binding energy by about $1.5 \mathrm{eV}$, as expected for $\mathrm{Sn}^{2+}$ configuration. This is not observed in our study both for polycrystalline and single-crystalline samples. The same conclusion is obtained from $\mathrm{Sn} 3 d_{5 / 2}$ levels [Figs. 2(e) and 2(f)] measured at $485.45 \mathrm{eV} \mathrm{BE}$, which is close to its value in the $\mathrm{Sn}$ monochalcogenides (485.7 eV in $\mathrm{SnS}$ and $\mathrm{SnSe}$, and $485.6 \mathrm{eV}$ in $\mathrm{SnTe}$ ).

The results of the above core-level PE experiments prove the extraordinary quality of our single-crystalline samples that are highly suitable for angle-resolved PE studies of the electronic structure of $\mathrm{Co}_{3} \mathrm{Sn}_{2} \mathrm{~S}_{2}$ presented below.

\section{B. Calculated band structure}

In the present study, the band structure of $\mathrm{Co}_{3} \mathrm{Sn}_{2} \mathrm{~S}_{2}$ was calculated using the approach and the crystalline parameters as described above. The obtained theoretical data are in accordance with the previous calculations of Weihrich and Anusca. $^{7}$

Our results both for the spin-resolved and spin-integrated band structures as well as for corresponding valence-band

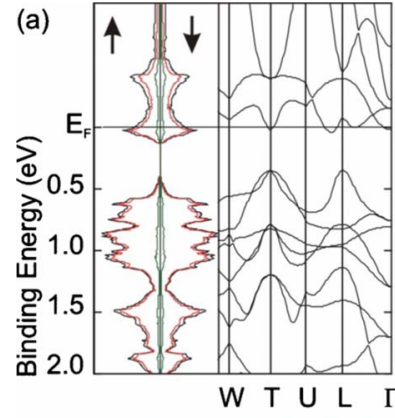

(c)

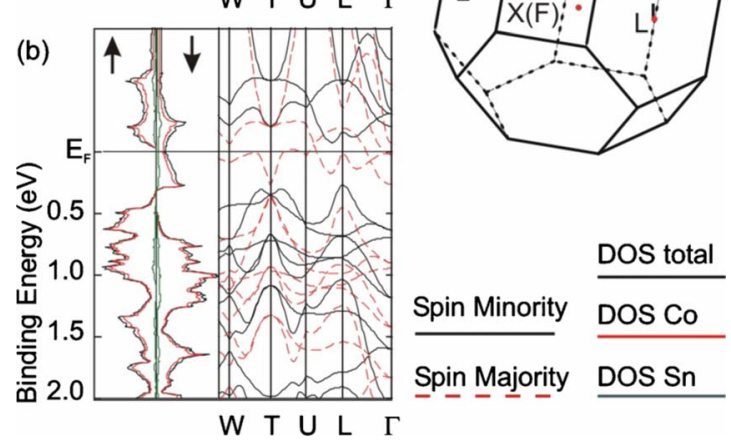

FIG. 3. (Color online) Electronic structure calculated for bulk $\mathrm{Co}_{3} \mathrm{Sn}_{2} \mathrm{~S}_{2}$ with (a) unpolarized and (b) polarized spins. (c) Brillouin zone of the compound. Rhombohedral axis intersects the border of the $\mathrm{BZ}$ at the $\mathrm{L}$ point while (111) axis crosses it at the $\mathrm{T}$ point. The surface projection of the $\mathrm{BZ}$ is constructed according to the hexagonal description of the unit cell.

density of electronic states are shown in Fig. 3. An energy gap between 0.15 and $0.45 \mathrm{eV} \mathrm{BE}$ is seen in the nonspinpolarized calculation. The metallic character of $\mathrm{Co}_{3} \mathrm{Sn}_{2} \mathrm{~S}_{2}$ is driven by bands crossing the Fermi energy above this energy gap. In a spin-polarized calculation, these bands are split due to exchange interaction. The spin-minority subbands occur above the Fermi energy while the spin-majority ones shift toward higher binding energies. As a result, the compound becomes insulating for the spin-minority channel while it remains metallic for the spin-majority direction. The bands around the gap are formed predominantly by Co $3 d$ states with a small admixture of $\mathrm{Sn}$ and $\mathrm{S}$ contributions. Noticeable $5 s$ and $5 p$ contributions from the $\mathrm{Sn}$ atoms lie between 5 and $7 \mathrm{eV}$ binding energy. Sn $4 d$ states are calculated at about 26 $\mathrm{eV} \mathrm{BE}$ for both inequivalent positions of these atoms. The calculated magnetic moment is $1.0 \mu_{B}$ per f.u., an integer value as expected for half-metallic ferromagnets. ${ }^{1}$

\section{Valence-band photoemission}

\section{Angle-integrated experiments}

Angle-integrated investigation of the VB electronic structure of $\mathrm{Co}_{3} \mathrm{Sn}_{2} \mathrm{~S}_{2}$ was performed by means of photoelectron spectroscopy with two different photon energies, $h \nu=21.2$ and $40.8 \mathrm{eV}$, in order to distinguish in PE signals the contributions of electronic states with different angular-momentum character. While at $h \nu=21.2 \mathrm{eV}$ cross sections of photoionization of Co $3 d$, and valence-band $p$ states of $\mathrm{Sn}$ and $\mathrm{S}$ are characterized by similar values; at $h \nu=40.8 \mathrm{eV}$ the Co $3 d$ states must cause much higher intensity. ${ }^{28}$ The obtained ex- 


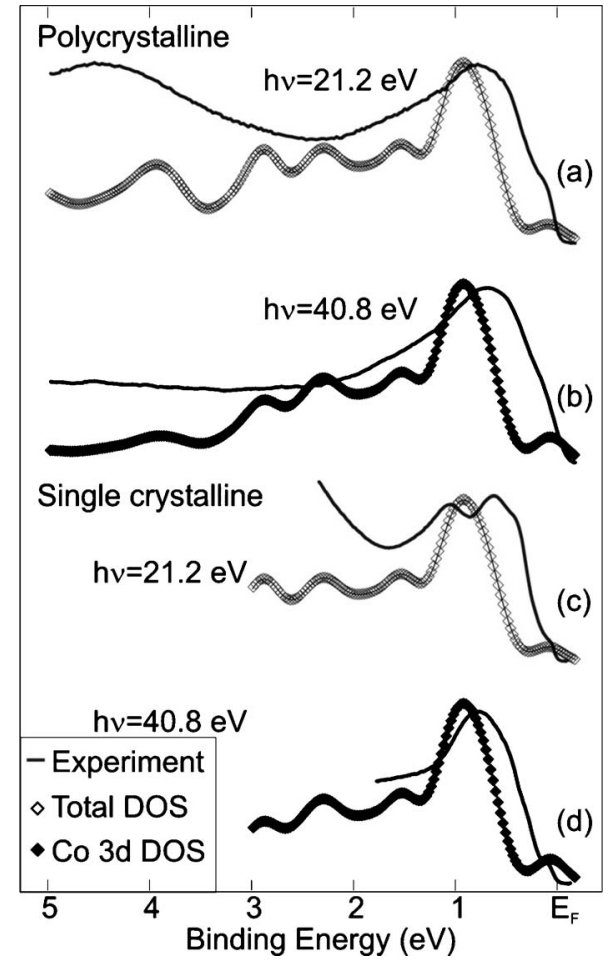

FIG. 4. Angle-integrated valence-band PE spectra of [(a) and (b)] polycrystalline and [(c) and (d)] single-crystalline samples [solid lines] compared with the calculated DOS convoluted with a Gaussian (150 meV, FWHM) (diamonds).

perimental results are compared with our band-structure calculations applying the LSDA formalism.

Polycrystals reveal no specific surface orientation. Thus, in contrast to single crystals, photoemission of polycrystalline samples provides results that are averaged over different surfaces. Therefore for a given compound, surface specific features can be derived by comparing PE data taken on both types of species. Valence-band spectra acquired for the polycrystalline $\mathrm{Co}_{3} \mathrm{Sn}_{2} \mathrm{~S}_{2}$ for 21.2 and $40.8 \mathrm{eV}$ photon energies are shown in Figs. 4(a) and 4(b). The experimental data depicted in Figs. 4(c) and 4(d) are obtained by integration over all measured directions and angles of the angle-resolved spectra taken for single-crystalline samples at the same photon energies. In this figure, the experimental results are compared to the calculated total and Co $3 d$-projected DOS, convoluted with a Gaussian (150 meV, FWHM), which simulates the finite experimental resolution.

The cross section of the $\mathrm{S} 3 p$ states drops drastically when going from 21.2 to $40.8 \mathrm{eV}$ photon energy as indicated by the change in the photoemission intensity in the range below $3 \mathrm{eV}$ of BE [compare Figs. 4(a) and 4(b)]. At the same time, the cross section of the Co $3 d$ states grows by almost one order of magnitude. Given that independently on photon energy the experimental spectra presented in Fig. 4 are characterized by resembling line shapes, only marginal contribution of the $\mathrm{S} 3 p$ states into the measured energy region of the valence bands is proven then experimentally, in agreement with the theory (Fig. 3). The main partial contribution here stems from the Co $3 d$ states.

Note that (i) the integrated spectra in Figs. 4(c) and 4(d) do not cover as much momentum $(\mathbf{k})$ space as the spectra

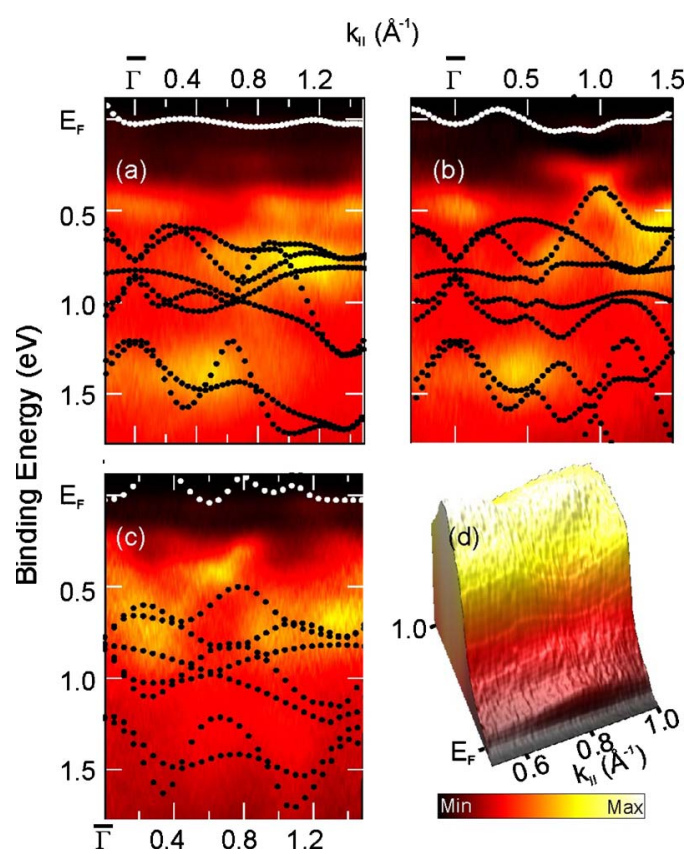

FIG. 5. (Color online) Angle-resolved PE spectra taken at $h \nu$ $=21.2 \mathrm{eV}$ along the (a) $\bar{\Gamma}-\bar{M}$ and (b) $\bar{\Gamma}-\bar{K}$ directions as well as at $h \nu=40.8 \mathrm{eV}$ along the $\bar{\Gamma}-\bar{M}$ (c) direction $\left(\mathbf{k}_{\|}-\right.$momentum vector parallel to the sample surface). (d) 3D color plot of PE intensity. Theoretical bands are shown by black (high BE) and white $\left(E_{F}\right)$ dots.

obtained from the polycrystalline samples, and (ii) measurements at $h \nu=21.2$ and $40.8 \mathrm{eV}$ were performed on different samples in order to probe each time freshly prepared sample surfaces that became contaminated after acquisition of each particular spectrum. Thus, distinct surfaces in both singlecrystalline measurements cannot be completely ruled out. This might be reflected by the appearance of a double-peak PE structure between 0.4 and $1.3 \mathrm{eV} \mathrm{BE}$ in Fig. 4(c) that is not found in Fig. 4(d), and is not present in the calculated DOS. Apart from this deviation and the finding that the main maxima of the experimental energy distribution curves lie closer to $E_{F}$, the experimental data are in general agreement with the calculated DOS. The fact that the main PE peak is located in the close vicinity of the Fermi energy complicates experimental observation of the energy gap predicted by the theory. The gap between the main peak and the Fermi-energy shoulder can be inferred, however, even from the polycrystalline data, suggesting its formation is not due to only one particular surface termination.

\section{Angle-resolved experiments}

A detailed assignment of the calculated nonmagnetic band structure to the angle-resolved PE spectra of $\mathrm{Co}_{3} \mathrm{Sn}_{2} \mathrm{~S}_{2}$ taken above $T_{C}$ at room temperature is given in Figs. 5 and 6. The theoretical bands (black and white dots) superimpose the experimental plots (color scale representation) in order to give an estimate for the width of the energy gap and the position of features appearing in the measurements. Three photon energies-21.2, 40.8, and $70 \mathrm{eV}$-were used to sample different regions in the BZ. Additionally, variable light polar- 


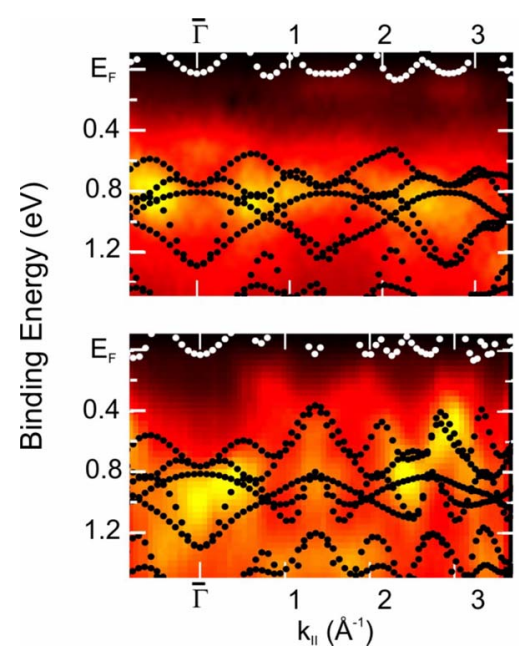

FIG. 6. (Color online) Angle-resolved PE spectra taken at $h \nu$ $=70 \mathrm{eV}$ along the $\bar{\Gamma}-\bar{M}$ direction with linear (top) horizontal and (bottom) vertical light polarizations. Theoretical bands are shown by black (high BE) and white $\left(E_{F}\right)$ dots.

ization (linear vertical and horizontal) was applied for $h \nu$ $=70 \mathrm{eV}$ in order to follow polarization dependent crosssection variations (see Fig. 6).

The momentum vector perpendicular to the surface $\left(\mathbf{k}_{\perp}\right)$ that is not conserved in photoemission has been evaluated from the free-electron model. ${ }^{29}$ Thereby the inner potential was first related to the very bottom of the calculated conduction bands and then adjusted to give best agreement with the measured spectra.

In spite of this rather simplified approach to the data interpretation, important correlation between the experimental and theoretical band dispersions can be concluded. As seen in Figs. 5 and 6 peaks of high intensity between 0.5 and 1.0 $\mathrm{eV}$ binding energy can be attributed to the dense bunches of the calculated bands. This correspondence was already inferred from our angle-integrated experiments. Note strong light-polarization dependence of the angle-resolved data in Fig. 6 reflecting bands of different symmetries for linear horizontal and vertical light polarizations. This shows that light with various polarizations should necessarily be used to map full electronic structure of $\mathrm{Co}_{3} \mathrm{Sn}_{2} \mathrm{~S}_{2}$ including information on the symmetry properties of the bands.

In Fig. 5, features below $0.5 \mathrm{eV}$ binding energy, and $\mathbf{k}_{\|}$ $=0.3,0.8$, and $1.0 \AA^{-1}$, respectively, can be particularly well described by the theory. It should be admitted that, however, there are some experimental observations that cannot be fully understood based on our single-particle LSDA groundstate calculations and the free-electron-like final-state model. Namely, the measured energy gap is much smaller than the predicted one and additional PE signals appear above the lower edge of the gap. Electron correlations that are not accounted for in our study might be responsible for the observed discrepancies.

As seen in the three-dimensional (3D) plot in Fig. 5(d) the PE intensity drops drastically when measuring from $0.25 \mathrm{eV}$ $\mathrm{BE}$ toward the Fermi level. The signal remaining at $E_{F}$ might be attributed to a background of scattered electrons. In this case, however, it should appear with nearly constant intensity (a)
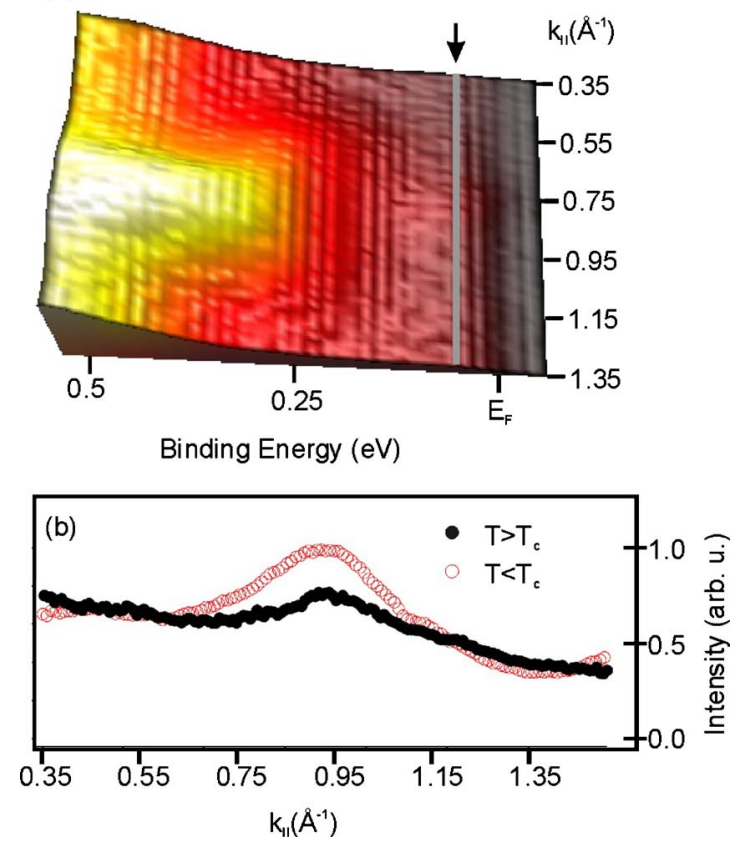

FIG. 7. (Color online) (a) 3D plot of angle-resolved PE intensity close to $E_{F}$ taken with $h \nu=21.2 \mathrm{eV}$ along the $\bar{\Gamma}-\bar{K}$ direction in the close vicinity of $40 \mathrm{~K}$. (b) Momentum distribution curves measured at the Fermi energy above (solid dots) and below (open dots) the Curie temperature (see text).

independently of the region probed in the Brillouin zone. Instead, a detailed data inspection yields weak Fermi-energy intensity modulations at different $\mathbf{k}_{\|}$. In order to increase the contrast, suffering from the low intensity at the Fermi level, the measured spectral line shapes were deconvoluted with a Gaussian accounting for the finite experimental resolution. By this procedure the Fermi-energy intensity modulations are emphasized and can be assigned to the Fermi-level crossings by the electronic bands that are predicted by our calculations (see Fig. 3).

Despite considerable surface sensitivity of the photoemission experiments, our study reveals good agreement between the measured data and the results of the bulk electronic structure calculations. This conclusion is similar to those drawn from photoemission investigations of high-temperature superconductors where bulk calculations were successfully used for angle-resolved data interpretation as well. ${ }^{30}$ This nontrivial finding might be related to the fact that the extended Bloch functions, which describe the bulk electronic states, penetrate with their trailing tails also in the surface region of solids and, therefore, can be probed in photoemission. In any case, the agreement between our experimental results and the calculations at room temperature make us confident to apply PE in order to verify changes in the electronic structure at $E_{F}$ at temperatures below $T_{C}(176.9 \mathrm{~K})$ predicted by the theory (see Fig. 3).

Corresponding angle-resolved PE spectra taken with $h \nu$ $=40.8 \mathrm{eV}$ at $T \sim 40 \mathrm{~K}$ in the range of energies up to $0.5 \mathrm{eV}$ below $E_{F}$ are depicted in Fig. 7(a). In Fig. 7(b) the momentum distribution of the PE intensity along the gray line [marked by arrow in Fig. 7(a)] is shown by open dots. In this 
plot the low-temperature $\mathbf{k}_{\|}$distribution curve (open dots) is compared with the curve acquired at room temperature (solid dots). Since the main differences between results calculated for $\mathrm{Co}_{3} \mathrm{Sn}_{2} \mathrm{~S}_{2}$ with unpolarized and polarized spins are seen close to $E_{F}$ (Fig. 3), the experimental data in Fig. 7(b) were normalized with each point to the PE intensity of the corresponding energy distribution curve integrated between 0.5 and $5 \mathrm{eV}$ BE.

A Fermi-energy signal enhancement at $\mathbf{k}_{\|}=0.95 \AA^{-1}$ is clearly monitored in this figure. This result supports the scenario drawn up by our spin-resolved band-structure calculations. The spin degeneracy of the Fermi-energy bands that are predicted to stabilize the half-metallic ferromagnetism in $\mathrm{Co}_{3} \mathrm{Sn}_{2} \mathrm{~S}_{2}$ is lifted for $T<T_{C}$ : the resulting spin-majority band is shifted far below $E_{F}$ causing the increase in PE intensity shown in Fig. 7.

\section{CONCLUSIONS}

The electronic structure of polycrystalline and singlecrystalline shandite $\mathrm{Co}_{3} \mathrm{Sn}_{2} \mathrm{~S}_{2}$ was studied by means of angle-resolved and core-level PE at temperatures below and above the Curie temperature. The experimental data are compared with the LSDA band-structure calculations carried out both for the spin-unpolarized and spin-polarized electronic configurations. It is shown that the rather surface sensitive experimental data are reproduced by the bulk computation. The growth of the $E_{F}$ signal measured below $T_{C}$ is explained by the lifting of the spin degeneracy at the Fermi level and shifting downward of the spin-majority bands that were unoccupied in the nonmagnetic case. Although our present spin-integrated data give strong evidence in favor of the halfferromagnetic scenario, spin-resolved measurements are necessary to quantify the expected spin polarization of the electrons at the Fermi level in $\mathrm{Co}_{3} \mathrm{Sn}_{2} \mathrm{~S}_{2}$.

\section{ACKNOWLEDGMENTS}

This study was supported by the Deutsche Forschungsgemeinschaft (DFG) Project Nos. MO 1049/1-1 and RO 2460/ 3-1. We are indebted to U. Burkhardt for the metallographic investigation of the $\mathrm{Co}_{3} \mathrm{Sn}_{2} \mathrm{~S}_{2}$ crystals.
${ }^{1}$ R. A. de Groot, F. M. Mueller, P. G. van Engen, and K. H. J. Buschow, Phys. Rev. Lett. 50, 2024 (1983).

${ }^{2}$ S. A. Wolf, Science 294, 1488 (2001).

${ }^{3}$ G. L. Bona, F. Maier, M. Taborelli, E. Bucher, and P. H. Schmidt, Solid State Commun. 56, 391 (1985).

${ }^{4}$ D. Ristoiu, J. P. Nozieres, C. N. Borca, T. Komesu, H.-K. Jeong, and P. A. Dowben, Europhys. Lett. 49, 624 (2000).

${ }^{5}$ K. E. H. M. Hanssen, P. E. Mijnarends, L. P. L. M. Rabou, and K. H. J. Buschow, Phys. Rev. B 42, 1533 (1990).

${ }^{6}$ S. J. Jenkins and D. A. King, Surf. Sci. 501, L185 (2002).

${ }^{7}$ R. Weihrich and I. Anusca, Z. Anorg. Allg. Chem. 632, 1531 (2006).

${ }^{8}$ R. Weihrich, A. C. Stückl, M. Zabel, and W. Schnelle, Z. Anorg. Allg. Chem. 630, 1767 (2004).

${ }^{9}$ J. M. D. Coey and M. Venkatesan, J. Appl. Phys. 91, 8345 (2002).

${ }^{10}$ T. Kubodera, H. Okabe, Y. Kamihara, and M. Matoba, Physica B 378, 1142 (2006)

${ }^{11}$ R. Weihrich, I. Anusca, and M. Zabel, Z. Anorg. Allg. Chem. 631, 1463 (2005).

${ }^{12}$ M. Zabel, S. Wandinger, and K.-J. Range, Z. Naturforsch. B 34B, 238 (1979).

${ }^{13}$ WIN-FIT program, URL: http://www.physik.tu-dresden.de/et/ winfit/winfit-install.exe

${ }^{14}$ K. Koepernik and H. Eschrig, Phys. Rev. B 59, 1743 (1999).

${ }^{15}$ J. P. Perdew and Y. Wang, Phys. Rev. B 45, 13244 (1992).

${ }^{16} \mathrm{H}$. Eschrig, Optimized LCAO Method and the Electronic Structure of Extended Systems (Springer, New York, 1989).
${ }^{17}$ P. Vaqueiro and G. G. Sobany, Solid State Sci. 11, 513 (2009).

${ }^{18}$ P. Gütlich, K.-J. Range, C. Felser, C. Schultz-Münzenberg, W. Tremel, D. Walcher, and M. Waldeck, Angew. Chem., Int. Ed. 38, 2381 (1999).

${ }^{19}$ H. van der Heide, R. Hemmel, C. F. van Bruggen, and C. Haas, J. Solid State Chem. 33, 17 (1980).

${ }^{20}$ A. Galtayries and J. Grimblot, J. Electron Spectrosc. Relat. Phenom. 98-99, 267 (1999).

${ }^{21}$ D. Briggs and M. P. Seak, Practical Surface Analysis (SalleSauerländer, Frankfurt am Main, 1990).

${ }^{22}$ A. B. Mandale, S. Badrinarayanan, S. K. Date, and A. P. B. Sinha, J. Electron Spectrosc. Relat. Phenom. 33, 61 (1985).

${ }^{23}$ M. V. Yablonskikh, R. Berger, U. Gelius, R. Lizarraga, T. B. Charikova, E. Z. Kurmaev, and A. Moewes, J. Phys.: Condens. Matter 18, 1757 (2006).

${ }^{24}$ A. Umetani, E. Nagoshi, T. Kubodera, and M. Matoba, Physica B 403, 1356 (2008).

${ }^{25}$ R. Weihrich, F. Schappacher, R. Poettgen, H. Rosner, and W. Schnelle (unpublished).

${ }^{26}$ R. B. Shalvoy, G. B. Fischer, and P. J. Stiles, Phys. Rev. B 15, 1680 (1977).

${ }^{27}$ P. De Padova, M. Fanfoni, R. Larciprete, M. Mangiantini, S. Priori, and P. Perfetti, Surf. Sci. 313, 379 (1994).

${ }^{28}$ J. J. Yeh and I. Lindau, At. Data Nucl. Data Tables 32, 1 (1985).

${ }^{29}$ F. J. Himpsel, Appl. Opt. 19, 3964 (1980).

${ }^{30}$ A. Damascelli, Z. Hussain, and Z.-X. Shen, Rev. Mod. Phys. 75, 473 (2003). 